\title{
APPLICATIONS OF VARIATIONAL INEQUALITIES TO THE EXISTENCE THEOREM ON QUADRATURE DOMAINS
}

BY

\author{
MAKOTO SAKAI
}

\begin{abstract}
In this paper we shall study quadrature domains for the class of subharmonic functions. By using the theory of variational inequalities, we shall give a new proof of the existence and uniqueness theorem. As an application, we deal with Hele-Shaw flows with a free boundary and show that their two weak solutions, one of which was defined by the author using quadrature domains and the other was defined by Gustafsson [3] using variational inequalities, are identical with each other.
\end{abstract}

Introduction. In a previous paper [7], the author has defined the quadrature domains of positive measures for the class of subharmonic functions and studied their applications to complex function theory.

Let $\nu$ be a finite positive measure on the two-dimensional Euclidean space $\mathbf{R}^{2}$. Let $S L^{1}(\Omega)$ be the class of subharmonic functions in an open set $\Omega$ which are integrable with respect to the two-dimensional Lebesgue measure $m$. A nonempty open set $\Omega$ is called a quadrature domain of $\nu$ for class $S L^{1}$ if

(Qi) $\nu$ is concentrated in $\Omega$, namely, $\nu\left(\Omega^{c}\right)=0$, where $\Omega^{c}$ denotes the complement of $\Omega$,

(Qii) $\int_{\Omega} s^{+} d \nu<\infty$ and $\int_{\Omega} s d \nu \leqslant \int_{\Omega} s d m$ for every $s \in S L^{1}(\Omega)$, where $s^{+}=$ $\max \{s, 0\}$.

(Qiii) $m(\Omega)<\infty$.

Let us denote by $Q\left(\nu, S L^{1}\right)$ the class of all quadrature domains of $\nu$ for class $S L^{1}$. The class $Q\left(\nu, S L^{1}\right)$ may be empty. Let $W$ be an open set with finite area and let $f$ be a nonnegative bounded integrable function in $\mathbf{R}^{2}$ satisfying $f=0$ a.e. in $W^{c}$. If $\sup _{W} f<1$, then $Q\left(f m, S L^{1}\right)=\varnothing$. The class $Q\left(\chi_{W} m, S L^{1}\right)$ consists of all open sets $\Omega$ satisfying $\chi_{W}=\chi_{\Omega}$ a.e. in $\mathbf{R}^{2}$, where $\chi_{W}$ denotes the characteristic function of $W$, namely, $\chi_{W}(x)=1$ for $x \in W$ and $\chi_{W}(x)=0$ for $x \notin W$.

On the contrary, the author has already proved the following theorem (cf. [7, Theorem 3.7]):

THEOREM 1. Let $f$ be a bounded integrable function in $\mathbf{R}^{2}$ such that $f \geqslant 1$ a.e. in a connected open set $W$ with finite area, $f=0$ a.e. in $W^{c}$ and $\int f d m>m(W)$, then $Q\left(f m, S L^{1}\right) \neq \varnothing$ and there exists a minimum domain $\tilde{W}$ in $Q\left(f m, S L^{1}\right)$, namely, $\Omega \in Q\left(f m, S L^{1}\right)$ if and only if $\tilde{W} \subset \Omega$ and $m(\Omega \backslash \tilde{W})=0$.

Received by the editors October 5, 1981 and, in revised form, February 23, 1982.

1980 Mathematics Subject Classification. Primary 31A05, 31B05; Secondary 35A15.

Key words and phrases. Quadrature domains, variational inequalities, subharmonic functions, potentials. 
The main purpose of this paper is to give this theorem a new proof by using variational inequalities.

Recently, Gustafsson [3] has used variational inequalities to solve a moving boundary problem for Hele-Shaw flows. As a corollary, he has proved the existence of quadrature domains of a finite sum of positive point masses for the class of all complex-valued analytic integrable functions [3, Corollary 16.1].

To obtain the result, Gustafsson has used the fact that the boundaries of the above quadrature domains are algebraic curves, so this is a very special case in the theory of quadrature domains. In this paper, we shall deal with a general case stated as in the theorem.

1. Variational inequalities. In this section, we shall show our theorem for a special function $f$ by using variationial inequalities. We assume that $W$ is a bounded open set $\mathbf{R}^{2}$ and $f$ is a bounded integrable function with $f>1$ a.e. in $W$ and $f=0$ a.e. in $W^{c}$. The proof will be divided into four steps. Each step is given as a proposition.

For a real-valued bounded integrable function $g$ in $\mathbf{R}^{2}$ with compact support, we define the logarithmic potential $U^{g}$ of $g$ by

$$
U^{g}(y)=\int(-\log |x-y|) g(x) d m(x),
$$

where $|x-y|=\left(\sum_{j=1}^{2}\left(x_{j}-y_{j}\right)^{2}\right)^{1 / 2}, x=\left(x_{1}, x_{2}\right)$ and $y=\left(y_{1}, y_{2}\right)$. It is known that $U^{g}$ is of class $C^{1}$ in $\mathbf{R}^{2}$ and $\Delta U^{g}=-2 \pi g$ in the sense of distributions. First we shall show the following lemma:

Lemma 1. Let $\Omega \in Q\left(f m, S L^{1}\right)$. Then $\Omega$ is bounded.

Proof. Let $f_{1}$ be a nonnegative integrable function in $\mathbf{R}^{2}$ such that $f_{1} \geqslant 1$ a.e. in an open set $W_{1}$ and $f_{1}=0$ a.e. in $W_{1}^{c}$. Let $\Omega_{1}$ satisfy $m\left(\Omega_{1}\right)<\infty$ and

$$
\int_{W_{1}} s f_{1} d m \leqslant \int_{\Omega_{1}} s d m
$$

for every $s \in S L^{\infty}\left(W_{1} \cup \Omega_{1}\right)$, where $S L^{\infty}\left(W_{1} \cup \Omega_{1}\right)$ denotes the class of all bounded subharmonic functions in $W_{1} \cup \Omega_{1}$.

First we show that if $\Omega_{1}$ is a bounded open set with smooth boundary, then $W_{1}$ is contained in the bounded open set $G$ whose boundary is the outer boundary of $\Omega_{1}$. Assume $W_{1} \backslash G \neq \varnothing$. Then $\left(\partial W_{1}\right) \backslash \bar{G} \neq \varnothing$.

Choose a point $x_{0} \in\left(\partial W_{1}\right) \backslash \bar{G}$ and $r>0$ so that $\operatorname{Cap}\left(W_{1}^{c} \cap \overline{B\left(x_{0} ; r\right)}\right)>0$ and $\bar{G} \cap \overline{B\left(x_{0} ; r\right)}=\varnothing$, where $B\left(x_{0} ; r\right)=\left\{x \in \mathbf{R}^{2}|| x-x_{0} \mid<r\right\}$. Let $\mu$ be the equilibrium distribution of $E=W_{1}^{c} \cap \overline{B\left(x_{0} ; r\right)}$ and let $u$ be the conductor potential of $E$, namely,

$$
u(y)=\int_{E}(-\log |x-y|) d \mu(x) .
$$

Then $u$ is bounded from above and harmonic in $E^{c}$. Set $\alpha=\sup _{\bar{G}} u$ and $s=$ $\max \{u, \alpha\}-\alpha$. Then $s \in S L^{\infty}\left(W_{1} \cup \Omega_{1}\right), \int_{W_{1}} s f_{1} d m>0$ and $\int_{\Omega_{1}} s d m=0$. This contradicts (1) and hence $W_{1} \subset G$. 
Since $W$ is bounded, we can choose a ball $B$ centered at the origin and $M>1$ so that $f \leqslant M \chi_{B}$ a.e. in $\mathbf{R}^{2}$. Set $f_{1}=\chi_{\Omega}+M \chi_{B}-f$ and $W_{1}=\Omega \cup B$. Let $\Omega_{1}$ be a ball centered at the origin such that $m\left(\Omega_{1}\right)=M m(B)$. We shall show that $\Omega_{1}$ satisfies (1). Then, by the above argument, we see that $W_{1}=\Omega \cup B$ is contained in $G=\Omega_{1}$, namely, $\Omega$ is bounded.

To show that $\Omega_{1}$ satisfies (1), let $s \in S L^{\infty}\left(W_{1} \cup \Omega_{1}\right)$. Let $s^{*}$ be a function in $S L^{\infty}\left(W_{1} \cup \Omega_{1}\right)$ which is harmonic in $\Omega$, and satisfies $s \leqslant s^{*}$ in $\Omega$ and $s=s^{*}$ a.e. in $\Omega_{1} \backslash \Omega$; note here that $W_{1} \cup \Omega_{1}=\Omega \cup \Omega_{1}$. Then

$$
\int s^{*}\left(\chi_{\Omega}+M \chi_{B}-f\right) d m=\int s^{*} M \chi_{B} d m \leqslant \int_{\Omega_{1}} s^{*} d m .
$$

Subtracting $\int\left(s^{*}-s\right) \chi_{\Omega \cap \Omega_{1}} d m$ from both sides, we obtain

$$
\int_{W_{1}} s f_{1} d m \leqslant \int\left\{s^{*} \chi_{\Omega \backslash \Omega_{1}}+s \chi_{\Omega \cap \Omega_{1}}+s^{*}\left(M \chi_{B}-f\right)\right\} d m \leqslant \int_{\Omega_{1}} s d m .
$$

This completes the proof.

Proposition 1. Let $\Omega \in Q\left(f m, S L^{1}\right)$ and set $u=-1 /(2 \pi) U^{x_{\Omega}-f}$.Then $u$ and $\Omega$ satisfy

(i) $u \geqslant 0$ in $\mathbf{R}^{2}$,

(ii) $u=0$ in $\Omega^{c}$,

(iii) $\Delta u=\chi_{\Omega}-f$ in the sense of distributions.

Proof. Since $W$ and $\Omega$ are both bounded, $\chi_{\Omega}-f$ has a compact support. Hence $u$ is well defined and (iii) is evident.

For every $y \in \mathbf{R}^{2}, \log |x-y| \in S L^{1}(\Omega)$ and so

$$
U^{x_{\Omega}-f}(y)=\int_{W}(\log |x-y|) f d m(x)-\int_{\Omega} \log |x-y| d m(x) \leqslant 0 .
$$

Hence $u \geqslant 0$ in $\mathbf{R}^{2}$. If $y \notin \Omega$, then both $\log |x-y|$ and $-\log |x-y|$ belong to $S L^{1}(\Omega)$. Hence $u(y)=-1 /(2 \pi) U^{\chi_{\Omega}-f}(y)=0$.

Let $B$ be a large open ball centered at the origin such that $\bar{W} \subset B$, and let $g_{B}(x, y)$ be the Green function in $B$ of the Laplacian relative to the first boundary condition with pole at $y$.

Set

$$
\psi(y)=-\frac{1}{2 \pi} \int_{B} g_{B}(x, y)\left(f-\chi_{B}\right)(x) d m(x) .
$$

Then $\psi \in C^{1}(B)$ and $\psi$ can be extended onto a neighborhood of $\bar{B}$ so that the extension, we also write it by $\psi$, is of class $C^{1}$ in the neighborhood. It is easy to show that $\psi=0$ on $\partial B$ and $\Delta \psi=f-\chi_{B}$ in $B$ in the sense of distributions.

Let us denote by $H^{1}(B)$ the Sobolev space $H^{1,2}(B)$ with the norm

$$
\|u\|_{H^{1,2}(B)}=\sum_{0 \leqslant|\alpha| \leqslant 1}\left\|D^{\alpha} u\right\|_{L^{2}(B)}
$$

and denote by $H_{0}^{1}(B)$ the closure of $C_{0}^{\infty}(B)$ in the above norm. According to Poincarés inequality, it is well known that $\|\nabla u\|_{L^{2}(B)}$ is a norm equivalent to the 
above norm for $H_{0}^{1}(B)$. In what follows, we shall understand that $H_{0}^{1}(B)$ is the Hilbert space with the norm $\|u\|=\|\nabla u\|_{L^{2}(B)}$ (see, e.g. Kinderlehrer and Stampacchia [5, Chapter II, §4]). We note here that $\psi \in H_{0}^{1}(B)$.

Let us consider the following variational problem: Minimize $\|\mathrm{h}\|$ in the closed convex set $K=\left\{h \in H_{0}^{1}(B) \mid h \geqslant \psi\right.$ a.e. in $\left.B\right\}$. The extremal function $v(\psi)$ exists and is determined uniquely. It is easy to show that $v=v(\psi)$ can be characterized by

(Vi) $v \in K$,

(Vii) $\int_{B} \nabla(h-v) \nabla v d m \geqslant 0$ for every $h \in K$.

Proposition 2. If $u \in H_{0}^{1}(B)$ and an open subset $\Omega$ of $B$ satisfy

(i)' $u \geqslant 0$ a.e. in $B$,

(ii)' $u=0$ a.e. in $B \backslash \Omega$,

(iii) $\Delta u=\chi_{\Omega}-f$ in $B$ in the sense of distributions, then $v=u+\psi$ satisfies (Vi) and (Vii).

Proof. It is evident that (Vi) follows from (i)'. Since $\Delta v=\Delta u+\Delta \psi=\chi_{\Omega}-\chi_{B}$ $\in L^{2}(B)$, we have

$$
\int_{B} \nabla(h-v) \nabla v d m=-\int_{B}(h-v) \Delta v d m=\int_{B \backslash \Omega}(h-v) d m
$$

for every $h \in H_{0}^{1}(B)$. The condition (Vii) follows from the following equalities:

$$
\int_{B \backslash \Omega}(h-v) d m=\int_{B \backslash \Omega}\{(h-\psi)-u\} d m=\int_{B \backslash \Omega}(h-\psi) d m .
$$

Proposition 3. If $v \in H_{0}^{1}(B)$ satisfies (Vi) and (Vii), then $u=v-\psi \in C^{1}(\bar{B})$ and $u=0$ on $\partial B$. The function $u$ and $\Omega=\{x \in B \mid u(x)>0\}$ satisfy (i)' to (iii)' in Proposition 2.

Proof. The condition (i)' follows from (Vi).

Since $\psi \in H_{0}^{1}(B)$ and $\Delta \psi=f-\chi_{B} \in L^{\infty}(B), \psi \in H^{2, s}(B)$ for every $s$ with $1<s<\infty$ (see, e.g. Kinderlehrer and Stampacchia [5, Chapter II, Theorem 4.10]). Hence $v \in H^{2, s}(B) \cap C^{1, \lambda}(\bar{B})$ for every $s$ with $2<s<\infty$, where $\lambda=1-2 / s$ (cf. e.g. [5, Chapter IV, Theorem 2.3]). Hence $u=v-\psi \in C^{1}(\bar{B})$ and $u=0$ on $\partial B$. Set $\Omega=\{x \in B \mid u(x)>0\}$. Then (ii)' is satisfied evidently.

Let $\rho$ be a function of class $C_{0}^{\infty}$ with $0 \leqslant \rho \leqslant 1$ in $B$. Since $v \pm \rho u \in K$ and $\Delta v \in L^{2}(B)$, by (Vii), we have

$$
\int_{B} \rho u \Delta v d m=\int_{B} \nabla(-\rho u) \nabla v d m=0
$$

for every $\rho$. Hence $u \Delta v=0$ a.e. in $B$ and so $\Delta u+\Delta \psi=\Delta v=0$ a.e. in $\Omega$. This implies that $\Delta u=1-f$ a.e. in $\Omega$.

On $I=B \backslash \Omega$, by definition, $u=0$ and so $\Delta u=0$ a.e. (see, e.g. [5, Chapter II, Appendix A, Lemma A4]). By (Vii), we have

$$
-\int \rho \Delta v d m \geqslant 0
$$

for every $\rho \in H_{0}^{1}(B)$ with $\rho \geqslant 0$. Hence $\Delta v \leqslant 0$ a.e. in $B$ and so $f-\chi_{B}=\Delta \psi=\Delta v$ $\leqslant 0$ a.e. on $I$. This implies that $m(W \backslash \Omega)=0$ since $f>1$ a.e. in $W$. Hence $\Delta u=0=-f$ a.e. on $I$. Combining this with $\Delta u=1-f$ a.e. in $\Omega$, we obtain (iii)'. 
LemMa 2. Let $\Omega$ be an open set stated as in Proposition 3. Then we can choose a large open ball $B$ so that $\bar{\Omega} \subset B$.

Proof. Take a ball $B_{0}$ and $M>1$ so that $f \leqslant M \chi_{B_{0}}$. Then it is easily verified that $Q\left(M \chi_{B_{0}} m, S L^{1}\right)$ consists of the ball $B_{1}$ which satisfies $m\left(B_{1}\right)=M m\left(B_{0}\right)$ and has the same center as $B_{0}$ (see $\left.[7, \S 1]\right)$. Choose a ball $B$ so that $\bar{B}_{1} \subset B$ and fix it.

As before Proposition 2, let us consider the obstacle problem and write $\psi=\psi(f)$, $K=K(f)$ and $v=v(f)$. For the corresponding function and the open set stated as in Proposition 3, we write $u=u(f)$ and $\Omega=\Omega(f)$, respectively. Then, by Propositions 1 and $2, \Omega\left(M \chi_{B_{0}}\right)=B_{1}$. Hence it is sufficient to show that if $f \leqslant f_{1}$, then $u(f) \leqslant u\left(f_{1}\right)$.

First we show that if $h \in K(f)$ and $\Delta h \leqslant 0$ a.e. in $B$, then $v(f) \leqslant h$ a.e. in $B$. Set $w=h-v(f)$. Then, as we have seen in the proof of Proposition $3, \Delta v(f)=0$ a.e. in $\Omega$. Hence $\Delta w=\Delta h \leqslant 0$ a.e. in $\Omega$ and so $w$ is superharmonic in $\Omega$. Since $w=h-\psi(f) \geqslant 0$ a.e. in $B \backslash \Omega$ and $w \in H_{0}^{1}(B)$, we have $w \geqslant 0$ a.e. in $B$, namely, $v(f) \leqslant h$ a.e. in $B$.

Now we shall show that if $f \leqslant f_{1}$, then $u(f) \leqslant u\left(f_{1}\right)$. Let $h=u\left(f_{1}\right)+\psi(f)$. Then $h \in K(f)$ and $\Delta h=\Delta u\left(f_{1}\right)+\Delta \psi(f) \leqslant \Delta u\left(f_{1}\right)+\Delta \psi\left(f_{1}\right)=\Delta v\left(f_{1}\right) \leqslant 0$ a.e. in $B$. Hence, by the above argument, we see that $u(f)+\psi(f)=v(f) \leqslant h=u\left(f_{1}\right)+$ $\psi(f)$. Therefore $u(f) \leqslant u\left(f_{1}\right)$. This completes the proof.

Proposition 4. If $u \in H_{0}^{1}(B)$ and an open set $\Omega$ with $\bar{\Omega} \subset B$ satisfy (i)' to (iii)' in Proposition 2, then $u \in C^{1}(\bar{B})$, and $\tilde{W}=\{x \in B \mid u(x)>0\}$ is the minimum open set in $Q\left(f m, S L^{1}\right)$.

Proof. The function $u(x)+1 /(2 \pi) \int_{B} g_{B}(y, x)\left(\chi_{\Omega}-f\right)(y) d m(y)$ belongs to $H_{0}^{1}(B)$ and is harmonic to $B$. This implies that it is identically equal to zero and so $u(x)=-1 /(2 \pi) \int_{B} g_{B}(y, x)\left(\chi_{\Omega}-f\right)(y) d m(y)$. Since $\bar{W} \cup \bar{\Omega} \subset B$, by (iii)',

$$
\begin{aligned}
\int_{B}\left\{g_{B}(y, x)\right. & \left.-\log \frac{1}{|y-x|}\right\}\left(\chi_{\Omega}-f\right)(y) d m(y) \\
= & -\int_{B} \nabla\left\{g_{B}(y, x)-\log \frac{1}{|y-x|}\right\} \nabla u(y) d m(y) .
\end{aligned}
$$

The above is equal to

$$
\int_{B} \Delta\left\{g_{B}(y, x)-\log \frac{1}{|y-x|}\right\} u(y) d m(y),
$$

because $u \in H_{0}^{1}(B)$. Since $g_{B}(y, x)-\log (1 /|y-x|)$ is harmonic, the above integral is equal to zero. Hence $u=-1 /(2 \pi) U^{\chi_{\Omega}-f}, u \in C^{1}(\bar{B})$ and $u \geqslant 0$ in $B$.

Set $\tilde{W}=\{x \in B \mid u(x)>0\}$. Then, by (i) ${ }^{\prime}$ and (ii)', we have $\chi_{\tilde{W}} \leqslant \chi_{\Omega}$ a.e. in $B$. Since $\Delta u=0$ a.e. in $B \backslash \tilde{W}$ (see, e.g. Kinderlehrer and Stampacchia [5, Chapter II, Appendix A, Lemma A4]) and $f>1$ a.e. in $W$, by (iii)', we see that $\chi_{W \cup \Omega} \leqslant \chi_{\tilde{W}}$ a.e. in $B$. Hence $\chi_{\tilde{W}}=X_{\Omega}$ a.e. in $B$.

Next let us show $\tilde{W} \in Q\left(f m, S L^{1}\right)$. In what follows, for the sake of simplicity, set $g=\chi_{\tilde{W}}-f$. Let $y \in B \backslash \tilde{W}$. Then $u(y)=0$. Since $u$ is of class $C^{1}$ and $u$ attains its minimum at $y, \partial u / \partial x_{j}(y)=0, j=1,2$. Hence $U^{g}=-2 \pi u=0$ and $\partial U^{g} / \partial x_{j}=$ $-2 \pi \partial u / \partial x_{j}=0$ in $B \backslash \tilde{W}$. 
Let $\left\{\omega_{n}\right\}_{n=1}^{\infty}$ be a sequence of $C^{\infty}$-functions in $\tilde{W}$ such that $0 \leqslant \omega_{n} \leqslant 1, \omega_{n}=0$ in a neighborhood of $\partial \tilde{W}, \omega_{n}=1$ outside a neighborhood of $\partial \tilde{W}, \lim _{n \rightarrow \infty} \omega_{n}(x)=1$ for all $x=\left(x_{1}, x_{2}\right) \in \tilde{W}$, and

$$
\left|D^{\alpha} \omega_{n}(x)\right| \leqslant A_{\alpha} n^{-1} \delta(x)^{-|\alpha|}\left(\log \frac{1}{\delta(x)}\right)^{-1}
$$

for all $x \in \tilde{W}$ and all multi-indices $\alpha$, where $A_{\alpha}$ denotes a constant depending only on $\alpha$, and $\delta(x)$ denotes the minimum of $e^{-2}$ and the distance from $x$ to $\partial \tilde{W}$. For the existence of the above sequence $\left\{\omega_{n}\right\}$, see Hedberg [4, p. 13, Lemma 4].

It follows that

$$
\begin{gathered}
\frac{\partial^{2}}{\partial x_{j}^{2}}\left(U^{g} \omega_{n}\right)=\frac{\partial^{2} U^{g}}{\partial x_{j}^{2}} \omega_{n}+2 \frac{\partial U^{g}}{\partial x_{j}} \frac{\partial \omega_{n}}{\partial x_{j}}+U^{g} \frac{\partial^{2} \omega_{n}}{\partial x_{j}^{2}}, \\
\Delta U^{g}=\sum_{j} \frac{\partial^{2}}{\partial x_{j}^{2}} U^{g}=-2 \pi g
\end{gathered}
$$

in the sense of distributions. Since

$$
\frac{\partial U^{g}}{\partial x_{j}}(x)-\frac{\partial U^{g}}{\partial x_{j}}(y)=o\left(|x-y| \log \frac{1}{|x-y|}\right), \quad j=1,2,
$$

for every pair of points $x$ and $y$ with $|x-y|<e^{-2}$,

$$
\begin{aligned}
U^{g}(x) & =o\left(\delta^{2}(x) \log \frac{1}{\delta(x)}\right), \\
\frac{\partial U^{g}}{\partial x_{j}}(x) & =o\left(\delta(x) \log \frac{1}{\delta(x)}\right), \quad j=1,2,
\end{aligned}
$$

in a neighborhood of each boundary point of $\tilde{W}$. Hence

$$
\int_{\tilde{W}} s g d m=\lim _{n \rightarrow \infty} \int_{\tilde{W}} s g \omega_{n} d m=-\frac{1}{2 \pi} \lim _{n \rightarrow \infty} \int_{\tilde{W}} s \Delta\left(U^{g} \omega_{n}\right) d m
$$

for every $s \in L^{1}(\tilde{W})$. If $s$ is subharmonic in $\tilde{W}$, then $\Delta s \geqslant 0$ in the sense of distributions. Let $\varphi$ be a nonnegative $C_{0}^{\infty}$-function of $|x|$ in $\mathbf{R}^{2}$ such that $\int \varphi d m=1$ and set $s_{\varepsilon}(x)=\int s(x-\varepsilon y) \varphi(y) d m(y)$ for $\varepsilon>0$. Then $s_{\varepsilon}$ is a subharmonic $C^{\infty}$ function on a given compact subset of $\tilde{W}$ for every sufficiently small $\varepsilon>0$, and $s_{\varepsilon} \downarrow s$ as $\varepsilon \downarrow 0$ on the compact set. Since $U^{g}=-2 \pi u \leqslant 0$, by letting $\varepsilon$ tend to 0 , we see that

$$
\int_{\tilde{W}} s g d m \geqslant 0
$$

for every $s \in S L^{1}(\tilde{W})$. Hence $\tilde{W} \in Q\left(f m, S L^{1}\right)$. Let $\Omega \in Q\left(f m, S L^{1}\right)$. Then, by Proposition 1 and the above argument, we see that $\chi_{\Omega}=\chi_{\tilde{w}}$ a.e. in $\mathbf{R}^{2}$. If $y \notin \Omega$, then $-\log |x-y| \in S L^{\prime}(\Omega)$ and so

$$
0 \leqslant \int(-\log |x-y|)\left(\chi_{\Omega}-f\right)(x) d m(x)=-2 \pi u(y) .
$$

Hence $u(y)=0$, namely, $y \notin \tilde{W}$. Therefore $\tilde{W} \subset \Omega$ for every $\Omega \in Q\left(f m, S L^{1}\right)$. The proof is now complete. 
Thus we have proved our theorem for the function $f$ given at the beginning of this section. From (2), we have an additional result which is also true for the function $f$ as in Theorem 2.

Corollary. Let $\Omega \in Q\left(f m, S L^{1}\right)$ and $s \in S L^{1}(\Omega)$. Then

$$
\int_{W} s f d m=\int_{\Omega} s d m
$$

if and only if $s$ is harmonic in $\tilde{W}$.

2. Proof of the theorem. In this section, we assume that $W$ is an open set in $\mathbf{R}^{2}$ with finite area and $f$ is a bounded integrable function with $f \geqslant 1$ a.e. in $W, f=0$ a.e. in $W^{c}$ and $\int_{O} f d m>m(O)$ for every connected component $O$ of $W$. We shall show the following as our main theorem:

THeOrem 2. Let $f$ and $W$ be as above. Then $Q\left(f m, S L^{1}\right) \neq \varnothing$ and there exists a minimum domain $\tilde{W}$ in $Q\left(f m, S L^{1}\right)$.

First we show the following two lemmas:

LEMMA 3. Let $f_{i}, i=1,2$, be bounded integrable functions in $\mathbf{R}^{2}$ such that $f_{i} \geqslant 1$ a.e. in open sets $W_{i}$ and $f_{i}=0$ a.e. in $W_{i}^{c}$, and let $\Omega_{i} \in Q\left(f_{i} m, S L^{1}\right), i=1,2$. If $f_{1} \leqslant f_{2}$ a.e. in $\mathbf{R}^{2}$, then $\chi_{\Omega_{1}} \leqslant \chi_{\Omega_{2}}$ a.e. in $\mathbf{R}^{2}$.

Proof. Assume that $\Omega_{1} \backslash \Omega_{2} \neq \varnothing$. Take a point $y \in \Omega_{1} \backslash \Omega_{2}$ and set

$$
s(x)= \begin{cases}g_{\Omega_{1}}(x, y) & \text { in } \Omega_{1}, \\ 0 & \text { in } \Omega_{2} \backslash \Omega_{1},\end{cases}
$$

where $g_{\Omega_{1}}(x, y)$ denotes the Green function in $\Omega_{1}$ with pole at $y$. Then $s \geqslant 0$ in $\Omega_{2} \cup \Omega_{1},-s \mid \Omega_{1} \in S L^{1}\left(\Omega_{1}\right)$ and $s \mid \Omega_{2}=s^{*}$ a.e. in $\Omega_{2}$ for some $s^{*} \in S L^{1}\left(\Omega_{2}\right)$, because $m\left(\Omega_{1}\right) \leqslant m\left(\Omega_{2}\right)<\infty$. Hence

$$
\int_{\Omega_{1}} s d m \leqslant \int s f_{1} d m \leqslant \int s f_{2} d m \leqslant \int_{\Omega_{2}} s d m
$$

and so

$$
\int_{\Omega_{1}, \Omega_{2}} s d m \leqslant \int_{\Omega_{2} \cup \Omega_{1}} s d m=0
$$

This implies that $m\left(\Omega_{1} \backslash \Omega_{2}\right)=0$, namely, $\chi_{\Omega_{1}} \leqslant \chi_{\Omega_{2}}$ a.e. in $\mathbf{R}^{2}$.

COROLlaRY. Let $f$ be a bounded integrable function in $\mathbf{R}^{2}$ such that $f \geqslant 1$ a.e. in an open set $W$ and $f=0$ a.e. in $W^{c}$. Let $\Omega_{i} \in Q\left(f m, S L^{1}\right), i=1,2$. Then $\chi_{\Omega_{1}}=\chi_{\Omega_{2}}$ a.e. in $\mathbf{R}^{2}$.

LEMMA 4. Let $g$ be a bounded nonnegative integrable function in $\mathbf{R}^{2}$ with compact support which is contained in a connected open set $W$. Let $\int g d m>0$ and $K$ be a compact subset of $W$. Then there are a bounded nonnegative integrable function $f_{g, K}$ in $\mathbf{R}^{2}$ and a bounded connected open set $W_{g, K}$ such that $f_{g, K}>0$ in $W_{g, K}, f_{g, K}=0$ in $W_{g, K}^{c}, K \cup \operatorname{supp} g \subset W_{g, K} \subset \overline{W_{g, K}} \subset W$ and $\int s g d m \leqslant \int s f_{g, K} d m$ for every $s \in$ $S L^{\mathrm{i}}(W)$. 
Proof. We may assume that $\inf _{x \in L} g(x)>0$ for a compact subset $L$ of $W$ with $m(L)>0$. Let $\delta$ be a number such that $0<\delta<d(L, \partial W) / 2$, where $d(L, \partial W)$ denotes the distance between $L$ and $\partial W$, and define a bounded nonnegative integrable function $g_{1}$ in $\mathbf{R}^{2}$ by

$$
g_{1}(x)=\int_{B(x ; \delta)} g(y) \chi_{L}(y) d m(y) / m(B(x ; \delta)) .
$$

Then $g_{1}$ is continuous, supp $g_{1}$ is compact and $\int s g d m \leqslant \int s\left(g \chi_{L^{c}}+g_{1}\right) d m$ for every $s \in S L^{1}(W)$. Take a ball $B_{1}$ and a number $\alpha_{1}>0$ so that $\overline{B_{1}} \subset W$ and $g_{1} \geqslant \alpha_{1}$ in $B_{1}$. For every $x \in\left(K \cup \operatorname{supp} g \cup \operatorname{supp} g_{1}\right)$, we can find balls $B_{j}, j=2,3, \ldots, n$, with centers $p_{j}$ such that $p_{n}=x, \bar{B}_{j} \subset W$ and $p_{j} \in B_{j-1}$ for every $j$. Let $\nu_{1}=\alpha_{1} \chi_{B_{1}}$. Assume that there are a bounded nonnegative integrable function $\nu_{j-1}$ in $\mathbf{R}^{2}$ and a number $\alpha_{j-1}>0$ such that $\operatorname{supp} \nu_{j-1} \subset \cup_{i=1}^{j-1} \bar{B}_{i}, \nu_{j-1} \geqslant \alpha_{j-1}$ in $\cup_{i=1}^{j-1} B_{i}$ and $\int s \nu_{j-1} d m \geqslant \int s \nu_{1} d m$ for every $s \in S L^{1}(W)$. Take a ball $B$ with center $p_{j}$ such that $B \subset B_{j-1} \cap B_{j}$. Then

$$
\begin{aligned}
\int s \nu_{j-1} d m & =\int s\left(\nu_{j-1}-\alpha_{j-1} \chi_{B}\right) d m+\alpha_{j-1} \int_{B} s d m \\
& \leqslant \int s\left(\nu_{j-1}-\alpha_{j-1} \chi_{B}\right) d m+\alpha_{j-1} \frac{m(B)}{m\left(B_{j}\right)} \int_{B_{j}} s d m
\end{aligned}
$$

for every $s \in S L^{1}(W)$. Set $\nu_{j}=\nu_{j-1}-\alpha_{j-1} \chi_{B}+\left(\alpha_{j-1} m(B) / m\left(B_{j}\right)\right) \chi_{B_{j}}$ and $\alpha_{j}=$ $\alpha_{j-1} m(B) / m\left(B_{j}\right)$. The function $\nu_{j}$ and a number $\alpha_{j}$ satisfy the above conditions for $j$. Thus, by induction, we can construct $\nu_{n}$ and $\alpha_{n}>0$ such that $\operatorname{supp} \nu_{n} \subset \cup_{j=1}^{n} \bar{B}_{j}$, $\nu_{n} \geqslant \alpha_{n}$ in $\cup_{j=1}^{n} B_{j}$ and $\int s \nu_{n} d m \geqslant \int s \nu_{1} d m$ for every $s \in S L^{1}(W)$.

Let us write $\nu_{x}$ and $V_{x}$ for $\nu_{n}$ and $\cup_{j=1}^{n} B_{j}$, respectively. Since $K \cup \operatorname{supp} g \cup \operatorname{supp} g_{1}$ is compact, we can find a finite number of open sets $V_{x_{1}}, \ldots, V_{x_{k}}$ such that $\left(K \cup \operatorname{supp} g \cup \operatorname{supp} g_{1}\right) \subset \cup_{j=1}^{k} V_{x_{j}}$. Set

$$
f_{g, K}=g \chi_{L^{c}}+g_{1}-\alpha_{1} \chi_{B_{1}}+\frac{1}{k} \sum_{j=1}^{k} \nu_{x_{j}}, \quad W_{g, K}=\bigcup_{j=1}^{k} V_{x_{j}} .
$$

These satisfy the required condition.

Proof of Theorem 2. At first, let us construct an open set $G \in Q\left(f m, S L^{1}\right)$. For every connected component $O_{i}$ of $W$, let $L_{i}$ be a compact subset of $O_{i}$ such that $\int(f-1) \chi_{L_{i}} d m>0$. Let $g_{i}=(f-1) \chi_{L_{i}}$ and let $\left\{O_{i, j}\right\}$ be an exhaustion of $O_{i}$ such that $\overline{O_{i, j}}$ is compact for every $j$. By using Lemma 4, we can find $f_{i, j}=f_{g_{i} / 2^{j}, \overline{o_{i, j}}}$ and $W_{i, j}=W_{g_{i} / 2^{j}, \overline{o_{i, j}}}$ such that $f_{i, j}>0$ in $W_{i, j}, f_{i, j}=0$ in $W_{i, j}^{c}, \overline{O_{i, j}} \cup L_{i} \subset W_{i, j} \subset \overline{W_{i, j}} \subset$ $W$ and $\int s g_{i} / 2^{j} d m \leqslant \int s f_{i, j} d m$ for every $s \in S L^{1}(W)$. Set

$$
f_{0}=f-\sum_{i=1}^{\infty} g_{i}, \quad f_{n}=f_{0} \chi_{W_{n}}+\sum_{1 \leqslant i \leqslant n} \sum_{1 \leqslant j \leqslant n-i+1} f_{i, j}, \quad n=1,2, \ldots,
$$

where $W_{n}=\cup_{1<i<n} \cup_{1<j<n-i+1} W_{i, j}$. Then $f_{n}$ is a bounded integrable function in $\mathbf{R}^{2}$ with $f_{n}>1$ in a bounded open set $W_{n}$ and $f_{n}=0$ in $W_{n}^{c}$.

From the argument given in $\S 1$, we can construct the minimum open set $\tilde{W}_{n} \in Q\left(f_{n} m, S L^{1}\right)$ for every $n$. Since $f_{n} \leqslant f_{n+1}$, from the proof of Lemma 2 , we 
obtain $u\left(f_{n}\right) \leqslant u\left(f_{n+1}\right)$ (for the notation, see the proof of Lemma 2). Hence $\tilde{W}_{n} \subset \tilde{W}_{n+1}$. Set $G=\bigcup \tilde{W}_{n}$. By the proof of Proposition 3, we have $m\left(W_{n} \backslash \tilde{W}_{n}\right)=0$. Hence it follows that $m(W \backslash G)=0$.

Next let us show

$$
\int s f d m \leqslant \int_{G} s d m
$$

for every $s \in S L^{1}(G)$. For every $\varepsilon>0$, we can take a number $n$ so that

$$
\int_{G} s d m+\varepsilon \geqslant \int_{\tilde{W}_{n}} s d m
$$

and

$$
\int s f d m-\varepsilon \leqslant \int s\left(f_{0} \chi_{W_{n}}+\sum_{1 \leqslant i \leqslant n} \sum_{1 \leqslant j \leqslant n-i+1} g_{i} / 2^{j}\right) d m
$$

Since

$$
\begin{aligned}
\int s\left(f_{0} \chi_{W_{n}}+\sum_{1 \leqslant i \leqslant n} \sum_{1 \leqslant j \leqslant n-i+1} g_{i} / 2^{j}\right) d m & \leqslant \int s\left(f_{0} \chi_{W_{n}}+\sum_{1 \leqslant i \leqslant n} \sum_{1 \leqslant j \leqslant n-i+1} f_{i, j}\right) d m \\
& \leqslant \int_{W_{n}} s f_{n} d m \leqslant \int_{\tilde{W}_{n}} s d m,
\end{aligned}
$$

we have

$$
\int s f d m \leqslant \int_{G} s d m+2 \varepsilon
$$

for every $\varepsilon>0$. Hence

$$
\int s f d m \leqslant \int_{G} s d m
$$

for every $s \in S L^{1}(G)$.

For $s=1$, we have

$$
\begin{aligned}
\int f d m & =\lim _{n \rightarrow \infty} \int\left(f_{0} \chi_{W_{n}}+\sum_{1 \leqslant i \leqslant n} \sum_{1 \leqslant j \leqslant n-i+1} g_{i} / 2^{j}\right) d m \\
& =\lim _{n \rightarrow \infty} \int f_{n} d m=\lim _{n \rightarrow \infty} m\left(\tilde{W}_{n}\right)=m(G) .
\end{aligned}
$$

Hence $m(G)<\infty$. Thus we have proved that $G \in Q\left(f m, S L^{1}\right)$.

From the corollary to Lemma $3, \chi_{\Omega}=\chi_{G}$ a.e. for every $\Omega \in Q\left(f m, S L^{1}\right)$. Since $\chi_{\Omega}-f$ has not necessarily compact support, take two distinct points $\zeta_{1}$ and $\zeta_{2}$ in $(\cup \Omega)^{c}$, where $\cup \Omega$ denotes the union of all $\Omega \in Q\left(f m, S L^{1}\right)$, consider the generalized logarithmic potential $U^{\chi_{\Omega}-f}\left(x ; \zeta_{1}, \zeta_{2}\right)$ (see $\left.[8, \S 3]\right)$ and set

$$
u(x)=-\frac{1}{2 \pi} U^{x_{\Omega}-f}\left(x ; \zeta_{1}, \zeta_{2}\right) \text {. }
$$

The function $u$ is determined independently of the choice of $\Omega \in Q\left(f m, S L^{1}\right)$. Let $\tilde{W}=\left\{x \in \mathbf{R}^{2} \mid u(x)>0\right\}$. If $\Omega \in Q\left(f m, S L^{1}\right)$ and $x \notin \Omega$, then $u(x)=0$ and so 
$x \notin \tilde{W}$. Therefore $\tilde{W} \subset \Omega$ for every $\Omega \in Q\left(f m, S L^{1}\right)$. Since $u(x)=0$ in $\tilde{W}^{c}, \Delta u=0$ a.e. on $\tilde{W}^{c}$. Hence $0=\Delta u=\chi_{\Omega}-f$ a.e. in $\tilde{W}^{c}$ and so $\chi_{\Omega} \leqslant \chi_{\tilde{W}}$ a.e. in $\mathbf{R}^{2}$. This implies that $\tilde{W} \subset \Omega$ and $m(\Omega \backslash \tilde{W})=0$ for every $\Omega \in Q\left(f m, S L^{1}\right)$.

Finally, the fact that $\tilde{W} \in Q\left(f m, S L^{1}\right)$ follows from the similar argument given in the proof of Proposition 4. In contrast with the proof of Proposition 4, the open set $\tilde{W}$ is not necessarily bounded. For the generalized logarithmic potential and the similar argument given in the proof of Proposition 4, see [8, §3].

3. The case of higher dimensions. Our theorem is also valid for the case of higher dimensions. In the case of dimension $d \geqslant 3$, let us write by $S_{d}$ the surface area of the $(d-1)$-dimensional unit hypersphere, namely, $S_{d}=2 \pi^{d / 2} / \Gamma(d / 2)$. We replace $-\log |x-y|$ by $|x-y|^{2-d}$ and consider the Newton potential

$$
U^{g}(y)=\int|x-y|^{2-d} g(x) d m(x)
$$

instead of the logarithmic potential which we have used in the case of dimension 2. In the above definition, $g$ is a real-valued bounded integrable function defined in $\mathbf{R}^{d}$ and $m$ denotes the $d$-dimensional Lebesgue measure.

It is known that

(1) $U^{g}$ is of class $C^{1}$,

(2) $\partial U^{g}(x) / \partial x_{j}-\partial U^{g}(y) / \partial x_{j}=O(|x-y| \log (1 /|x-y|)), j=1,2, \ldots, d$, for every pair of points $x$ and $y$ with $|x-y|<e^{-2}$.

(3) $\Delta U^{g}=-(d-2) S_{d} g$ in the sense of distributions.

Therefore our arguments are also valid if we replace $-1 /(2 \pi)$ and $-\log |x-y|$ by $-1 /\left((d-2) S_{d}\right)$ and $|x-y|^{2-d}$, respectively.

Let us give here a remark on the generalized logarithmic potential used in the proof of Theorem 2. It is unnecessary to consider "generalized" in the case of dimension $d \geqslant 3$. Because we can define the Newton potential $U^{g}$ of a bounded integrable function $g$ which has not necessarily a compact support.

4. Hele-Shaw flows with a free boundary. As an application of the new proof of our theorem, we deal with Hele-Shaw flows with a free boundary produced by the injection of fluid into the narrow gap between two parallel planes (for the mathematical formulation, see Richardson [6] and Sakai [7]).

In [7], the author has defined a weak solution of a free boundary problem of Hele-Shaw flows with the initial connected open set $\Omega(0)$. It is a family $\{\Omega(t)\}_{t \geqslant 0}$ of quadrature domains $\Omega(t)$ such that $\Omega(t)$ is the minimum domain in $Q\left(\chi_{\Omega(0)} m+\right.$ $\left.t \delta_{c}, S L^{1}\right)$ for every $t>0$, where $\delta_{c}$ denotes the Dirac measure at the injection point $c \in \Omega(0)$ of the fluid.

Recently, Gustafsson [3] has defined another weak solution of Hele-Shaw flows by using variational inequalities (for the case having the container wall, see Elliott and Janovský [2]).

Let $f_{t}=\chi_{\Omega(0)}+t(1 / m(B(c ; r))) \chi_{B(c ; r)}$ (in [3], Gustafsson has used $2 \pi t$ and $B(0 ; r)$ for $t$ and $B(c ; r)$, respectively), where $\Omega(0)$ denotes a bounded connected open set and $B(c ; r)$ satisfies $\overline{B(c ; r)} \subset \Omega(0)$, and consider the variational problem given before Proposition 2 for large ball $B_{t}$ (which depends on $t$ ) and for a function 
$\psi_{t}=\psi\left(f_{t}\right)$. Then Gustafsson's weak solution $\{\Omega(t)\}_{t \geqslant 0}$ is, in our notation given in the proof of Lemma 2, a family of domains $\Omega(t)=\Omega(0) \cup \Omega\left(f_{t}\right)$ for every $t>0$.

In this section, we shall note first that $\Omega(t)=\Omega\left(f_{t}\right)$, namely, $\Omega(0) \subset \Omega\left(f_{t}\right)$ (this result is also given by Gustafsson [3, Lemma 14(iv)]) and next show that the above two weak solutions are identical with each other.

The first assertion follows immediately from the following lemma:

LEMMA 5. Let $f, W$ and $\tilde{W}$ be as in Theorem 2. Then $W \subset \tilde{W}$.

Proof. Since $f \geqslant 1$ a.e. in $W, \Delta u=\chi_{\tilde{W}}-f \leqslant 0$ a.e. in $W$. Hence $u$ is a nonnegative superharmonic function in $W$. If $u(x)=0$ for some $x \in W$, then $u \equiv 0$ in the connected component of $W$ containing $x$. This contradicts $m(W \backslash \tilde{W})=0$ and so $u(x)>0$ in $W$, namely, $W \subset \tilde{W}$.

The next corollary guarantees that $\Omega\left(f_{t}\right)$ is connected.

COROLlary. If $W$ is connected, then $\tilde{W}$ is also connected.

Proof. Assume that $\tilde{W}$ is disconnected. Since $W \subset \tilde{W}$ and $W$ is connected, we can find a connected component $O$ of $\tilde{W}$ such that $W \cap O=\varnothing$. For every $s \in S L^{1}(\tilde{W} \backslash O)$, let $\tilde{s}$ be a function defined by $\tilde{s}(x)=s(x)$ in $\tilde{W} \backslash O$ and $\tilde{s}(x)=0$ in $O$. Then $\tilde{s} \in S L^{1}(\tilde{W})$ and

$$
\int_{W} s f d m=\int_{W} \tilde{s} f d m \leqslant \int_{\tilde{W}} \tilde{s} d m=\int_{\tilde{W} \backslash O} s d m .
$$

Hence $\tilde{W} \backslash O \in Q\left(f m, S L^{1}\right)$. This contradicts the fact that $\tilde{W}$ is the minimum domain in $Q\left(f m, S L^{1}\right)$.

To show the second assertion, by the argument given in $\$ 1$, it is sufficient to show that $Q\left(\chi_{\Omega(0)} m+t \delta_{c}, S L^{1}\right)=Q\left(f_{t} m, S L^{1}\right)$ for every $t>0$. This follows immediately from the proposition below.

For the sake of simplicity, we assume that $W$ is a connected open set. Let $\mu$ be a positive finite measure with compact support contained in $W$. For a number $\alpha$ with $0<\alpha<d(\operatorname{supp} \mu, \partial W) / 2$, where $d(\operatorname{supp} \mu, \partial W)$ denotes the distance between supp $\mu$ and $\partial W$, let us define a bounded function $M_{\alpha} \mu$ by

$$
\left(M_{\alpha} \mu\right)(x)=\frac{\mu(B(x ; \alpha))}{m(B(x ; \alpha))} .
$$

The support of $M_{\alpha} \mu$ is contained in $W$.

LEMMA 6. $Q\left(\chi_{W} m+\mu, S L^{1}\right)=Q\left(\left(\chi_{W}+M_{\alpha} \mu\right) m, S L^{1}\right)$.

Proof. We may assume that $\mu \neq 0$. If $\Omega \in Q\left(\left(\chi_{W}+M_{\alpha} \mu\right) m, S L^{1}\right)$, then, by Lemma $5, W \subset \Omega$. Since

$$
\int s d \mu \leqslant \int s\left(M_{\alpha} \mu\right) d m
$$

for every $s \in S L^{1}(W), \Omega \in Q\left(\chi_{W} m+\mu, S L^{1}\right)$. 
Conversely, assume that $\Omega \in Q\left(\chi_{W} m+\mu, S L^{1}\right)$. Set $G=\left\{x \in W \mid\left(M_{\alpha} \mu\right)(x)>\right.$ $0\}$. Then, since $M_{\alpha} \mu$ is lower semicontinuous, $G$ is an open set containing supp $\mu$. We shall show $\bar{G} \subset \Omega$. If $y \in \bar{G} \backslash \Omega$, then $\mu(B(y ; \beta))>0$ for $\beta$ with $\alpha<\beta<$ $d(\operatorname{supp} \mu, \partial W) / 2$. Set

$$
s(x)=\max \{\log (1 /|x-y|), \log (1 / \beta)\}-\log (1 / \beta) .
$$

Then $s \mid \Omega \in S L^{1}(\Omega)$. Since $m(W \backslash \Omega)=0$,

$$
\int s\left(\chi_{W} d m+d \mu\right)>\int_{W} s d m=\int_{\Omega} s d m
$$

This contradicts $\Omega \in Q\left(\chi_{W} m+\mu, S L^{1}\right)$. Hence $\bar{G} \subset \Omega$.

Let $s \in S L^{1}(\Omega)$, and let $s^{*} \in S L^{1}(\Omega)$ be harmonic in $G$ and satisfy $s^{*}=s$ a.e. in $\Omega \backslash G$. Since

$$
\int_{W} s^{*}\left(\chi_{W}+M_{\alpha} \mu\right) d m=\int_{W} s^{*}\left(\chi_{W} d m+d \mu\right) \leqslant \int_{\Omega} s^{*} d m
$$

and $s \leqslant s^{*}$ in $G$, we have

$$
\begin{aligned}
\int_{W} s\left(\chi_{W}+M_{\alpha} \mu\right) d m & \leqslant \int_{W} s^{*}\left(\chi_{W}+M_{\alpha} \mu\right) d m+\int_{G}\left(s-s^{*}\right) d m \\
& \leqslant \int_{\Omega} s^{*} d m+\int_{G}\left(s-s^{*}\right) d m=\int_{\Omega} s d m .
\end{aligned}
$$

Therefore $\Omega \in Q\left(\left(\chi_{W}+M_{\alpha} \mu\right) m, S L^{1}\right)$.

Proposition 5. Let $\mu_{i}, i=1,2$, be positive finite measures with compact support contained in a connected open set $W$. If there is an open subset $G$ of $W$ such that $G \supset \operatorname{supp} \mu_{1} \cup \operatorname{supp} \mu_{2}$ and $\int h d \mu_{1}=\int h d \mu_{2}$ for every harmonic function in $G$, then $Q\left(\chi_{W} m+\mu_{1}, S L^{1}\right)=Q\left(\chi_{W} m+\mu_{2}, S L^{1}\right)$.

Proof. By Lemma 6, it is sufficient to show that $Q\left(\left(\chi_{W}+M_{\alpha} \mu_{1}\right) m, S L^{1}\right)=$ $Q\left(\left(\chi_{W}+M_{\alpha} \mu_{2}\right) m, S L^{1}\right)$ for small $\alpha>0$. We obtain this equality by using Lemma 5 and the argument as in the proof of Lemma 6.

5. Quadrature domains for harmonic and analytic functions. In [7], quadrature domains for harmonic and analytic functions are introduced. Let $\nu$ be a positive finite measure in $\mathbf{R}^{2}$ and let $H L^{1}(\Omega)$ (resp. $A L^{1}(\Omega)$ ) be the class of all real-valued (resp. complex-valued) harmonic (resp. analytic) integrable functions in $\Omega$. A nonempty open set $\Omega$ is called a quadrature domain of class $H L^{1}$ (resp. $A L^{1}$ ), if $\Omega$ satisfies (Qi), (Qiii) and

$$
\int_{\Omega}|h| d \nu<\infty \text { and } \int_{\Omega} h d \nu=\int_{\Omega} h d m
$$

for every $h \in H L^{1}(\Omega)$ (resp. $\left.h \in A L^{1}(\Omega)\right)$. We denote by $Q\left(\nu, H L^{1}\right)\left(\right.$ resp. $\left.Q\left(\nu, A L^{1}\right)\right)$ the class of all quadrature domains of $\nu$ for class $H L^{1}$ (resp. $A L^{1}$ ). 
By using the generalized logarithmic potential, we obtain the following proposition:

Proposition 6. Let $f$ and $W$ be as in Theorem 2. Let $\Omega$ be an open set with finite area, let $\zeta_{1}$ and $\zeta_{2}$ be two distinct points in $\Omega^{c}$ and set $u(x)=-1 /(2 \pi) U^{x_{\Omega}-f}\left(x ; \zeta_{1}, \zeta_{2}\right)$. Then

(1) $\Omega \in Q\left(f m, S L^{1}\right)$ if and only if $u=0$ in $\Omega^{c}$ and $u \geqslant 0$ in $\Omega$,

(2) $\Omega \in Q\left(f m, H L^{1}\right)$ if and only if $u=0$ and $\partial u / \partial x_{j}=0, j=1,2$, in $\Omega^{c}$,

(3) $\Omega \in Q\left(f m, A L^{1}\right)$ if and only if $\partial u / \partial x_{j}=0, j=1,2$, in $\Omega^{c}$.

Proof. The assertions (1) and (2) are proved from the argument similar to the proof of Proposition 4. Let $\left(\chi_{\Omega}-f\right)^{\wedge}$ be the generalized Cauchy transform of $\chi_{\Omega}-f$ (for the definition, see [8]). Then $\left(\chi_{\Omega}-f\right)^{\hat{y}}=\left(\partial / \partial x_{1}-i \partial / \partial x_{2}\right) U^{\chi_{\Omega}-f}$. Hence $\partial u / \partial x_{j}=0, j=1,2$, in $\Omega^{c}$ implies that $\left(\chi_{\Omega}-f\right)^{\hat{n}}=0$ in $\Omega^{c}$. Let $z=x_{1}+i x_{2}$. Since the subclass of $A L^{1}(\Omega)$ which consists of all linear combinations of $1 /\left(z-\zeta_{k}\right)$ with $\zeta_{k} \in \Omega^{c}$ is dense in $A L^{1}(\Omega)$ (see Bers [1]), the assertion (3) follows.

\section{REFERENCES}

1. L. Bers, An approximation theorem, J. Analyse Math. 14 (1965), 1-4.

2. C. M. Elliott and V. Janovský, A variational inequality approach to Hele-Shaw flow with a moving boundary, Proc. Roy. Soc. Edinburgh Sect. A 88 (1981), 93-107.

3. B. Gustafsson, Applications of variational inequalities to a moving boundary problem for Hele Shaw flows, TRITA-MAT-1981-9, Mathematics, Roy. Inst. Tech., Stockholm, p. 84.

4. L. I. Hedberg, Approximation in the mean by solutions of elliptic equations, Duke Math. J. 40 (1973), 9-16.

5. D. Kinderlehrer and G. Stampacchia, An introduction to variational inequalities and their applications, Academic Press, New York, 1980.

6. S. Richardson, Hele Shaw flows with a free boundary produced by the injection of fluid into a narrow channel, J. Fluid Mech. 56 (1972), 609-618.

7. M. Sakai, Quadrature domains, Lecture Notes in Math., vol. 934, Springer-Verlag, Berlin, 1982.

8. __ Null quadrature domains, J. Analyse Math. 40 (1981), 144-154.

Department of Mathematics, Faculty of Science, Tokyo Metropolitan University, Fukazawa, SETAGaYA-KU, TOKYO, JAPAN 